Meta

Journal des traducteurs

Translators' Journal

\title{
Connaître Freud avant de le traduire
}

\section{Jean Laplanche et Jean Bertrand Pontalis}

Volume 27, numéro 1, mars 1982

Psychanalyse et traduction

URI : https://id.erudit.org/iderudit/003855ar

DOI : https://doi.org/10.7202/003855ar

Aller au sommaire du numéro

Éditeur(s)

Les Presses de l'Université de Montréal

ISSN

0026-0452 (imprimé)

1492-1421 (numérique)

Découvrir la revue

Citer cet article

Laplanche, J. \& Pontalis, J. (1982). Connaître Freud avant de le traduire. Meta, 27(1), 32-36. https://doi.org/10.7202/003855ar d'utilisation que vous pouvez consulter en ligne.

https://apropos.erudit.org/fr/usagers/politique-dutilisation/ 


\title{
CONNAÎTRE FREUD AVANT DE LE TRADUIRE*
}

\author{
Jean Laplanche et Jean-Bertrand Pontalis
}

Qui songerait à confier la traduction de Kant à un germaniste non philosophe, à un non-spécialiste? Certes, par rapport aux philosophes, Freud se trouve dans une position particulière: il ne se situe pas dans une tradition qui lui apporterait un appareil conceptuel et terminologique déjà constitué. Freud est un fondateur, il emprunte aux vocabulaires les plus différents : neurophysiologie, physique, psychologie de son temps, psychologie pathologique, philosophie, mythologie. Surtout il utilise toutes les ressources de l'allemand courant et les multiples combinaisons qu'il permet. Quand on dit que Freud est clair, on entend souvent que sa pensée s'accommode d'une terminologie peu rigoureuse, d'une expression à laquelle l'auteur lui-même n'attacherait qu'une importance secondaire : sentiment le plus souvent illusoire et auquel en tout cas le traducteur ne saurait se fier.

Les premières traductions de Freud en ont pâti. Pour faire élégant, coulant, voire rendre acceptable à nos «sensibilités latines» la «lourdeur germanique », on contourne les points obscurs, on cherche avec plus ou moins de bonheur les équivalents familiers : insensiblement la traduction glisse alors vers la transposition. Ceci souvent dans la meilleure intention du monde. L'ennui, quand on place au-dessus de tout le souci d'écrire en "bon français», c'est qu'on n'est bientôt plus en mesure d'apprécier le moment où l'on escamote une idée neuve.

Un exemple: Freud soutient, et c'est une thèse majeure, que le rêve est un «accomplissement de désir» (Wunscherfüllung). Le traducteur tombe sur la phrase qui, littéralement s'énonce : «deux accomplissements de désirs opposés viennent concourir dans une expression unique»; il va traduire, comme s'il craignait de se voir infliger «lourd» ou «mal-dit» dans la marge : «deux désirs opposés... s'accomplissent...» Nuance infime pour le profane mais qui ne peut manquer de retenir l'attention du psychanalyste : la locution substantive à elle seule n'indique-t-elle pas ici que l' «accomplissement de désir» n'est pas seulement une fonction mais une sorte de réalité psychique (un fantasme) susceptible de se combiner avec d'autres? Quitte à se montrer un peu rugueux, il faut bien respecter le substantif.

Vaut-il mieux alors sacrifier l'élégance et faire «technique»?

* Paru dans Le Monde $\mathrm{n}^{\circ} 6884$ du 1er mars 1967. 
Mais le simple emploi de majuscules (pour ne rien dire des néologismes) peut gauchir la compréhension.

C'est ainsi qu'une certaine tradition veut que les psychanalystes écrivent le «Moi» lorsqu'il s'agit de «l'instance» de la personnalité qu'a définie Freud. Or, dans le texte, aucune différence de graphie ne distingue un usage dit «technique » des nombreuses autres occurrences où se rencontre le terme «das Ich». $\mathrm{Au}$ nom de la rigueur technique, écrire tantôt moi, tantôt $M o i$, c'est en fait livrer la traduction à un préjugé théorique (on pourrait repérer sans conteste un usage non spécifique du terme «le moi») et refuser au chercheur travaillant sur la traduction française, la possibilité de dégager lui-même toutes les complexités de la théorie freudienne de la personnalité.

Le préjugé de l'élégance et le préjugé de la technicité (je sais mieux que Freud) se rejoignent pour réduire les arêtes et les détours du texte. Une traduction ne mérite pas la qualification de scientifique parce qu'elle se veut technique, encore moins si elle «en remet» quant à la technicité. Toute traduction suppose une connaissance méthodologique, une appropriation progressive de l'œuvre; a fortiori quand il s'agit, comme c'est le cas avec Freud (que les qualités de l'écrivain ne nous le fassent pas oublier!) d'une ouvre scientifique. Connaitre ici signifie d'abord se mouvoir dans la totalité de l'œuvre comme dans un milieu naturel: il faut être en mesure d'en dégager la cohérence et d'en apprécier les remaniements pour déterminer ensuite la fonction de tel élément, pour retrouver les ébauches successives d'une idée, pour reconnaître l'émergence d'un terme d'abord banal mais qui peu à peu devient le point où se nouent les fils jusqu'alors épars de la théorie.

C'est seulement alors qu'on peut poser certains problèmes plus particuliers. Soit le cas de doublets : deux termes de la langue allemande (le plus souvent l'un d'origine germanique, l'autre d'origine latine) fonctionnent à peu près comme synonymes; en français, un seul terme leur correspond dans la langue courante. Faut-il aller chercher un second terme insolite, ou même forger une locution ou un vocable nouveau afin de respecter une correspondance terme à terme? C'est là un problème qui n'admet pas de solution a priori. Ses données dépendent, bien sûr, des résonances, des implications de chacun des termes dans l'histoire et la civilisation allemandes. Mais sa solution est avant tout fonction de la façon dont l'auteur s'est ou non glissé dans le plan de clivage que lui offrait la langue, pour l'élargir éventuellement en opposition. Ainsi les mots Realität et Wirklichkeit; en français: réalité. Jean Hyppolite dans son admirable traduction de la Phénoménologie de l'esprit de Hegel, opte, à juste titre, pour deux traductions différentes : «réalité» et «réalité effective» (ou «effectivité"). Chez Freud, une enquête soigneuse révèle à l'inverse que les deux mots sont pratiquement interchangeables. Nous traduirons donc ici par un seul terme en français : réalité. Sans pour autant être fiers de notre exploit: nous savons combien Realität et Wirklichkeit résonnent différemment à l'oreille germanique. Entre deux maux (réduire à rien une nuance de la langue - imposer au lecteur français une fausse opposition conceptuelle) on choisit le moindre.

C'est dans le cadre de ce problème des doublets justiciables chaque fois d'une solution spécifique, que doit être replacée la trop fameuse question de 
l'instinct et de la pulsion. Question en passe de devenir un thème bien parisien depuis que le Lecteur-de-Freud (sic) a tonné devant un auditoire ébahi : Freud ne parle jamais d'instinct et il faut traduire Trieb par «dérive» (sic). Or tout lecteur de Freud, avec un petit 1 , sait qu'on trouve chez lui les deux termes Trieb et Instinkt. Le second, il est vrai, assez rarement, au point que JeanFrançois Revel dans le louable zèle de distinguer chez Lacan ce qui est lecture et ce qui est interprétation a été amené à forger le terme de Todesinstinkt et, par une sorte de fausse reconnaissance, à l'attribuer à Freud...

Mais laissons là ce combat à livres fermés. En allemand et dans la langue de Freud nous rencontrons deux termes, l'un d'origine germanique, Trieb, l'autre d'origine latine et française, Instinkt. Dans le français courant, un seul mot : instinct. Que nous apprend la philologie? L'enracinement de la notion de Trieb dans un mode de penser qui en fait une force relativement indéterminée, une énergie vitale qui pousse dans une certaine direction sans se fixer d'emblée un but précis et univoque. Instinkt, lui, conserve nécessairement de ses attaches étrangères l'écho d'une problématique plus spécifique : l'adaptation surprenante, la quasi-connaissance des moyens et des fins que révèlent certaines conduites spontanées. C'est le fameux «instinct des animaux» des cartésiens à Buffon et au-delà.

Qu'on ne méconnaisse pas cette prégnance du texte latin: dans la culture européenne des dix-huitième et dix-neuvième siècles le mot français représente la monnaie d'échange qui sert de référence.

Le germaniste omni-praticien ne peut guère avancer plus loin sans être ou devenir spécialiste d'un auteur ou d'un courant de pensée déterminé. Chez tel penseur, les deux notions en viennent à se recouvrir, tel autre donne une fonction au doublet, un troisième choisira de n'utiliser que l'un des deux termes, par exemple Trieb. Mais même dans ce dernier cas, le traducteur qui traduira Trieb par «instinct» devra rester conscient et avertir son lecteur, du glissement qu'il introduit par là, glissement d'autant plus sensible que l'auteur allemand en question possédait nécessairement dans son trésor personnel, le terme Instinkt auquel il a préféré celui de Trieb. Chez Freud la théorie du Trieb est depuis 1905 jusqu'à la fin au cœur même de la doctrine. Sans vouloir la résumer rappelons certains points essentiels : la notion d'une force biologique et psychique qualitativement indéterminée, la contingence des «objets», la variabilité et le polymorphisme des conduites dans lesquelles le Trieb va chercher à se satisfaire. À l'inverse, les occurrences d'Instinkt sont peu nombreuses. Dans un seul texte, Freud utilise comme des synonymes Herdentrieb et Herdeninstinkt, l'instinct grégaire. Mais il est alors lui-même traducteur: il discute un concept qui lui est étranger et qu'il se refuse à reprendre à son compte: the instinct of the herd de Trotter. Dans les autres passages où Freud utilise le terme Instinkt, c'est de façon concertée (le terme est d'ailleurs souligné). Il s'agit de l'instinct des animaux (Instinkt der Tiere), de leur «connaissance instinctive» (Instinktives Wissen des Tiere). Freud se demande alors, avec circonspection et sans répondre, s'il existe chez l'homme quelque chose d'analogue (Analoges) à cet Instinkt. Un philologue décrète ici «la cause est entendue », il s'agit de la même chose que du Trieb ! Pourquoi, dans ce cas, Freud poserait-il en 1915 une telle 
question? Il a centré toute sa théorie sur le Trieb depuis 1905 , et le voilà qui se demande si le Trieb existe bien et quelle conséquence cette hypothétique existence pourrait avoir chez l'homme? Question météore qui aurait été aussi vite oubliée que posée: jamais Freud ne remettra en cause l'existence du Trieb...

La véritable question de Freud (X, 294 et XII, 155-7) concerne, elle, l'existence chez l'homme de «formations» (Bildungen) cognitives et quasi cognitives (eine Art von schwer bestimbarem Wissen) qui préexisteraient chez l'enfant à toute expérience individuelle tout en le préparant à comprendre cette expérience (eine Vorbereitung zum Verständnis). On connaît la réponse ou plutôt l'hypothèse que Freud propose: il existerait des «fantasmes originaires », des «schèmes phylogénétiquement hérités» qui seraient, chez l'individu, la trace d'expériences faites par l'humanité au cours de sa préhistoire. Ce patrimoine «instinctif» (instinktiver Besitz - dieses Instinktive) «... serait le noyau de l'inconscient, une activité mentale primitive, qui plus tard est détrônée et recouverte par la raison humaine...» (XII, 166).

Concluons sur ce point : Freud a besoin ici du terme Instinkt, «instinct» en son sens classique, latin, afin d'indiquer la voie des structures mentales innées. Le Trieb, lui, dans son indétermination et son polymorphisme originaire, reste, comme poussée biologique, bien différent, et le vieux mot français de pulsion (dont le Robert fait remonter l'usage à 1625) n'est pas si mal choisi pour le traduire.

Dans quelle confusion la polémique fait tomber certains auteurs, on en jugera en constatant l'usage qui est fait de ce problème bien limité dans l'horrifique dispute qui oppose "biologistes» et «structuralistes». Comme si, en français, le terme de pulsion n'était pas adopté par les psychanalystes qui tiennent le plus à conserver au Trieb son assise biologique? Comme si, en anglais, le terme de drive (par différence avec celui d'instinct) n'était pas proposé par certains disciples orthodoxes de Freud et, qui plus est, par des disciples d'origine germanique! Comme si surtout, chez Freud, il était contestable que le Trieb soit une force biologique même si elle differe de ce qu'on nomme en français «instinct». Bien plus, s'il est vrai que Freud fait leur place chez l'homme à la fois au biologique et aux structures mentales ( «Schèmes", «formations ", «catégories" sont ses termes), c'est le Trieb, la pulsion, qui se situe du côté du biologique, tandis que la structure œdipienne, en tant qu'elle aurait un fondement héréditaire, est considérée comme ce qui est analogue (ce qui ne signifie pas : identique) à l'instinct animal.

La mode a monté en épingle cet exemple; mais cent autres montreraient aussi bien la nécessité d'une étude soigneuse et exhaustive du texte et de la pensée freudiens. S'il faut se battre sur des mots, qu'on sache au moins ce qu'ils désignent pour l'auteur qui les utilise. Comment, autrement, pourrait-on décider si tels termes quasi synonymes méritent ou non d'être codés dans la traduction, si telle particule verbale peut être ou non négligée?

A l'inverse du cas Trieb/Instinkt un même mot allemand peut recouvrer des significations que le français distingue. Angst, par exemple, est à la fois angoisse et peur. Fant-il alors, comme on le propose de façon irréfléchie, tra- 
duire "selon le contexte" (et par là ouvrir la porte à l'arbitraire du traducteur), ou bien admettre que Freud vise une unité notionnelle qui mérite d'être repérée dans la traduction soit par l'adoption d'un équivalent (angoisse) qui prend une valeur conventionnelle, soit par la mention, entre crochets, du terme allemand. Dans l'un et l'autre cas on renonce, nous objectera-t-on, à traduire. Mais tout traducteur n'est-il pas conscient de la difficulté et, à la limite, de l'impossibilité de sa tâche? Ne va-t-il pas parfois jusqu'à rêver, se détruisant ou s'accomplissant lui-même, que, pour être fidèle, il devrait purement et simplement recopier le texte original? C'est seulement une fois acquise la conscience aiguë qu'en toute rigueur aucun texte n'est traduisible (comme le démontre Georges Mounin qui pourtant n'aime guère "philosopher», au long des 297 pages de ses Problèmes théoriques de la traduction) que les vrais problèmes commencent à se poser. Tant qu' on en reste au plan des déclarations d'intention, il y a un consensus : chacun proteste qu'il donnera l'équivalent le plus exact possible du texte original, qu'il respectera jusqu'aux ambiguïtés et obscurités. C'est au lecteur d'interpréter, pas à lui! Bien sûr, mais avec ce vœu pieux, on n'a rien dit ou rien fait.

Il faut se colleter des années avec un auteur pour repérer les pièges innombrables qu'offre non seulement $l a$, mais $s a$ traduction. On ajoutera que ces pièges ne sont pas seulement d'ordre lexicologique: le meilleur germaniste, même muni d'un code exhaustif et raisonné des principaux concepts, risque de multiplier encore les erreurs et les contresens s'il n'est pas au sens plein et avant tout un «freudologue».

Tâche importante si elle doit aboutir à un document qui fasse foi, tâche écrasante pour un seul individu, la traduction de Freud peut, au premier chef bénéficier d'un travail d'équipe où se rejoignent psychanalystes, germanistes, philosophes, ceux qui de naissance, connaissent l'allemand (et le «viennois») et ceux qui manient de façon experte, toutes les ressources du français. Ces équipes visent non seulement à l'élaboration de traductions mais à la formation de conceptions scientifiques communes et à l'apprentissage de la rigueur ce qui doit leur permettre, le moment venu, d'essaimer en de nouveaux groupes. C'est de cette façon qu'il faut tenter de faire face à cette tâche «impossible» mais urgente. 